\title{
La fiscalidad de las cooperativas en Mexico
}

\author{
María Dolores Mejía Zarza1 \\ Universidad Autónoma del Estado de México (UAEM)
}

doi: http://dx.doi.org/10.18543/dec-16-2020pp 111-127

Recibido: 16.10.2020

Aceptado: 17.11.2020

Sumario: Introducción. I. Disposiciones Generales. II. Régimen fiscal de las cooperativas de producción a la luz del Impuesto sobre la Renta. III. Tratamiento fiscal de las cooperativas de producción. IV. Tratamiento fiscal para los socios que integran las cooperativas de producción. V. Régimen económico de las sociedades cooperativas. VI. Funcionamiento y administración de las sociedades cooperativas en México. VII. Conclusiones. VIII. Bibliografía.

Resumen. Con la reforma que se presenta, y que entrara en vigor a partir del próximo ejercicio fiscal, las Sociedades Cooperativas van a tener un verdadero régimen fiscal que las regule, debido a que sus actividades son propias del régimen general de personas morales las cuales deben de aplicar a futuro para el pago del impuesto del ejercicio como corresponde, esto es a través lo estipulado por el Artículo 10 de la propia Ley del Impuesto Sobre la Renta que se encuentra en vigor, y no así por lo dispuesto en el Artículo 177 que regula la forma de realizar el pago del impuesto como si se tratare del régimen de personas físicas.

Palabras clave: Nueva reforma fiscal, impuesto sobre la renta y empresas cooperativas.

Abstract. With the reform that is presented and that will come into force as of the next fiscal exercise, the Cooperative Societies will have a true fiscal regime that regulates them, because their activities are typical of the general regime of legal entities which must apply in the future for the payment of the tax for the fiscal exercise as deserved, this is through the provisions of Article 10 of the Income Tax Law itself that is in force, and not by the provisions of Article 177 that regulates the way to pay the tax as if it were the regime for natural persons.

Keywords: New fiscal reform, income tax and cooperative companies.

1 M. en D. Maestra titular de la materia de Derecho Fiscal en la Facultad de Derecho de la Universidad Autónoma del Estado de México (UAEM). 


\section{Introducción}

Las Sociedades Cooperativas en nuestro país, han venido funcionando desde hace ya varios años, bajo el régimen de personas morales que regula la Ley del Impuesto Sobre la Renta en su Título II; en el año 2005 surge una reforma a este título la cual entro en vigor a partir del mes de enero del año 2006, y se crea un apartado especial en el que se establece el régimen fiscal bajo el cual habrían de operar a partir de entonces, en el mes de septiembre del año en curso, se presenta el proyecto de reformas fiscales para el ejercicio fiscal del año 2014, mediante la cual se establece que estas Sociedades Cooperativas se manejen bajo el régimen general de personas morales, debido a que las actividades que realizan esta clase de Sociedades, cumple con todos los lineamientos a seguir por el Título II de la Ley del Impuesto Sobre la Renta; lo que explica la realización del presente artículo sobre la Fiscalidad de las Cooperativas en México.

\section{Disposiciones generales}

De acuerdo con la Ley General de Sociedades Cooperativas², en México encontramos tres clases de sociedades:

- De consumidores de bienes y/o servicios,

- De productores de bienes y/o servicios, y

- De ahorro y préstamo.

Son sociedades cooperativas de consumidores, aquéllas cuyos miembros se asocien con el objeto de obtener en común artículos, bienes y/o servicios para ellos, sus hogares o sus actividades de producción ${ }^{3}$, y podrán dedicarse a actividades de abastecimiento y distribución, así como a la prestación de servicios relacionados con la educación o la obtención de vivienda.

Son sociedades cooperativas de productores, aquéllas cuyos miembros se asocien para trabajar en común en la producción de bienes y/o servicios, aportando su trabajo personal, físico o intelectual. Independientemente del tipo de producción a la que estén dedicadas, estas sociedades podrán almacenar, conservar, transportar y comercializar sus productos, de acuerdo con lo establecido en la propia Ley ${ }^{4}$ General de Sociedades Cooperativas.

\footnotetext{
2 Artículo 21 LGSC

3 Artículo 22 LGSC

4 Artículo 27 LGSC
} 
Se consideran dos clases de categorías de sociedades cooperativas de producción 5 :

- Ordinarias, y

- De participación estatal. Para tal efecto, el Estado podrá dar en concesión o administración bienes o servicios a las sociedades cooperativas, en los términos que señalen las leyes respectivas.

Son sociedades cooperativas ordinarias, las que para funcionar requieren únicamente de su constitución legal; en tanto que las sociedades cooperativas de participación estatal, son aquellas que se asocian con autoridades federales, estatales, municipales o los órganos político-administrativos del Distrito Federal, para la explotación de unidades productoras o de servicios públicos, dados en administración, o para financiar proyectos de desarrollo económico a niveles local, regional o nacional.

Son sociedades cooperativas de ahorro y préstamo ${ }^{6}$, aquellas que de acuerdo con la Ley tengan por objeto la realización de estas actividades. Entendiéndose como ahorro la captación de recursos a través de depósitos de ahorro de dinero de sus Socios; y como préstamo, la colocación y entrega de los recursos captados entre sus mismos Socios.

\section{Régimen fiscal de las sociedades cooperativas de producción a la luz del impuesto sobre la renta}

A partir del $1 .^{\circ}$ De enero del año dos mil seis, se establece en nuestro país un nuevo régimen fiscal aplicable a las Sociedades Cooperativas de Producción, el cual me permito citar de manera textual, con el propósito de explicar de forma correcta como se encuentra reglamentado el régimen fiscal de estas sociedades, no sin antes hacer mención que este régimen de personas morales lo contempla y reconoce la Ley General de Sociedades Mercantiles en su Artículo 1.․

Artículo $10^{\circ}$-Esta Ley reconoce las siguientes especies de sociedades mercantiles:

I. Sociedad en nombre colectivo;

II. Sociedad en comandita simple;

III. Sociedad de responsabilidad limitada;

IV. Sociedad anónima;

6 Artículo 33 LGSC 
V. Sociedad en comandita por acciones, y

VI. Sociedad cooperativa.

De igual forma en su Artículo 212 la Ley General de Sociedades Mercantiles, señala que estas Sociedades Cooperativas se regirán por su propia Ley, esto es por la Ley General de Sociedades Cooperativas.

\section{SECRETARIA DE HACIENDA Y CREDITO PÚBLICO DECRETO por el que se adiciona un Capítulo VII-A al Título II de la Ley del Impuesto Sobre la Renta.}

Al margen un sello con el Escudo Nacional, que dice: Estados Unidos Mexicanos.- Presidencia de la República.

VICENTE FOX QUESADA, Presidente de los Estados Unidos Mexicanos, a sus habitantes sabed:

Que el Honorable Congreso de la Unión, se ha servido dirigirme el siguiente

\section{DECRETO}

«EL CONGRESO GENERAL DE LOS ESTADOS UNIDOS MEXICANOS, DECRETA:

\section{SE ADICIONA UN CAPÍTULO VII-A AL TÍTULO II DE LA LEY DEL IMPUESTO SOBRE LA RENTA.}

Artículo Único.-Se adiciona un Capítulo VII-A «De las Sociedades Cooperativas de Producción», que comprende los artículos 85-A y 85-B, al Título II de la Ley del Impuesto Sobre la Renta, para quedar como sigue:

\section{CAPÍTULO VII-A \\ De las Sociedades Cooperativas de Producción}

Artículo 85-A._Las sociedades cooperativas de producción que únicamente se encuentren constituidas por socios personas físicas, para calcular el impuesto sobre la renta que les corresponda por las actividades que realicen, en lugar de aplicar lo dispuesto en el Título II de esta Ley, podrán aplicar lo dispuesto en la Sección I del Capítulo II del Título IV de la misma, considerando lo siguiente:

I. Calcularán el impuesto del ejercicio de cada uno de sus socios, determinando la parte de la utilidad gravable del ejercicio que le corresponda a cada socio por su participación en la sociedad cooperativa de que se trate, aplicando al efecto lo dispuesto en el artículo 130 de esta Ley.

Las sociedades cooperativas de producción a que se refiere este Capítulo, podrán diferir la totalidad del impuesto a que se refiere esta fracción, hasta el ejercicio fiscal en el que distribuyan a sus socios la utilidad gravable que les corresponda. 
Cuando la sociedad cooperativa de que se trate distribuya a sus socios utilidades provenientes de la cuenta de utilidad gravable, pagará el impuesto diferido aplicando al monto de la utilidad distribuida al socio de que se trate la tarifa a que se refiere el artículo 177 de esta Ley.

Para los efectos del párrafo anterior, se considerará que las primeras utilidades que se distribuyan son las primeras utilidades que se generaron.

El impuesto que en los términos de esta fracción corresponda a cada uno de sus socios, se pagará mediante declaración que se presentará ante las oficinas autorizadas, a más tardar el 17 del mes inmediato siguiente a aquél en el que se pagaron las utilidades gravables, el socio de la cooperativa de que se trate podrá acreditar en su declaración anual del ejercicio que corresponda el impuesto que se pague en los términos de este párrafo.

Para los efectos de este Capítulo, se considerará que la sociedad cooperativa de producción distribuye utilidades a sus socios, cuando la utilidad gravable a que se refiere esta fracción se invierta en activos financieros diferentes a las cuentas por cobrar a clientes o en recursos necesarios para la operación normal de la sociedad de que se trate.

Para los efectos de este capítulo las sociedades cooperativas de producción que no distribuyan rendimientos a sus socios, solo podrán invertir dichos recursos en bienes que a su vez generan más empleos o socios cooperativistas;

II. Las sociedades cooperativas de producción llevarán una cuenta de utilidad gravable. Esta cuenta se adicionará con la utilidad gravable del ejercicio y se disminuirá con el importe de la utilidad gravable pagada.

El saldo de la cuenta prevista en esta fracción, que se tenga al último día de cada ejercicio, sin incluir la utilidad gravable del mismo, se actualizará por el periodo comprendido desde el mes en el que se efectuó la última actualización y hasta el último mes del ejercicio de que se trate. Cuando se distribuyan utilidades provenientes de esta cuenta con posterioridad a la actualización prevista en este párrafo, el saldo de la cuenta que se tenga a la fecha de la distribución, se actualizará por el periodo comprendido desde el mes en el que se efectuó la última actualización y hasta el mes en el que se distribuyan dichas utilidades.

El saldo de la cuenta de utilidad gravable deberá transmitirse a otra u otras sociedades en los casos de fusión o escisión. En este último caso, dicho saldo se dividirá entre la sociedad escindente y las sociedades escindidas, en la proporción en la que se efectúe la partición del capital contable del estado de posición financiera aprobado por la asamblea general extraordinaria y que haya servido de base para realizar la escisión. 
La utilidad gravable a que se refiere esta fracción, será la que determine la sociedad cooperativa de que se trate, en los términos del artículo 130 de esta Ley, correspondiente a la totalidad de los socios que integran dicha sociedad;

III. Por los ingresos que obtenga la sociedad cooperativa no se efectuarán pagos provisionales del impuesto sobre la renta, y

IV. Los rendimientos y los anticipos que otorguen las sociedades cooperativas a sus socios, se considerarán como ingresos asimilados a los ingresos por la prestación de un servicio personal subordinado y se aplicará lo dispuesto en los artículos 110 y 113 de esta Ley.

Artículo 85-B.-Las sociedades cooperativas de producción que opten por aplicar lo dispuesto en el presente Capítulo, no podrán variar su opción en ejercicios posteriores, salvo cuando se cumplan con los requisitos que se establezcan en el Reglamento de esta Ley. Cuando los contribuyentes dejen de pagar el impuesto en los términos de este Capítulo, en ningún caso podrán volver a tributar en los términos del mismo.

\section{Transitorios}

Artículo Primero.-El presente Decreto entrará en vigor el 10. de enero de 2006.

Artículo Segundo.-Para los efectos del tercero y cuarto párrafos de la fracción I, del artículo 85-A de la Ley del Impuesto Sobre la Renta, se pagará el impuesto sobre la renta conforme a la tarifa del artículo 177 de la citada Ley vigente al momento en el que se generaron las utilidades que se distribuyan.

Artículo Tercero.-Las sociedades cooperativas de producción que distribuyan anticipos o rendimientos a sus miembros en los términos de la fracción XI del artículo 29 de la Ley del Impuesto Sobre la Renta, para los efectos del artículo 9o. de la Ley del Impuesto al Activo, podrán considerar el impuesto sobre la renta que hubieren retenido por dichos conceptos conforme a lo dispuesto en el Capítulo I del Título IV de la Ley mencionada, como impuesto sobre la renta correspondiente a la sociedad cooperativa de que se trate.

Asimismo, las sociedades cooperativas de producción, para los efectos del acreditamiento a que se refiere el artículo 9o. de la Ley del Impuesto al Activo, será el que se determine conforme a lo dispuesto en la fracción I, del artículo 85-A de la Ley del Impuesto Sobre la Renta.

México, D.F., a 12 de septiembre de 2005. —Dip. Heliodoro Díaz Escárraga, Presidente.- Sen. Enrique Jackson Ramírez, Presidente. -Dip. Marcos Morales Torres, Secretario._- Sen. Micaela Aguilar González, Secretaria.- Rúbricas».

En cumplimiento de lo dispuesto por la fracción I del Artículo 89 de la Constitución Política de los Estados Unidos Mexicanos, y para su debida publicación y observancia, expido el presente Decreto en la 
Residencia del Poder Ejecutivo Federal, en la Ciudad de México, Distrito Federal, a los primer día del mes de diciembre de dos mil cinco.Vicente Fox Quesada. —Rúbrica._- El Secretario de Gobernación, Carlos María Abascal Carranza.- Rúbrica.

El primer párrafo del Artículo 85-A establece la opción para el pago del Impuesto Sobre la Renta para las Sociedades Cooperativas de Producción constituidas únicamente por socios personas físicas, por su parte la fracción I, hace mención al cálculo del impuesto de cada socio en términos del Artículo 130 de la propia Ley, esto es el impuesto se calculará por ejercicios fiscales de acuerdo con el Artículo 177 de la misma Ley del Impuesto Sobre la Renta; en la fracción II se hace referencia la cuenta de utilidad gravable que deben de llevar las sociedades cooperativas de producción, la cual será adicionada a la utilidad gravable del ejercicio; las fracciones III y IV, refieren que las sociedades cooperativas no están obligadas a realizar pagos provisionales del impuesto.

Por su parte el Artículo 85-B señala de manera estricta la prohibición de variar la opción de aplicar lo dispuesto en el capítulo VII-A para las sociedades cooperativas de producción, en ejercicios posteriores.

\section{Tratamiento fiscal de las sociedades cooperativas de producción}

Sección I del Capítulo II del Título IV Artículo 130 de la Ley del Impuesto Sobre la Renta

Sociedad Cooperativa $X$

Estado de Rendimientos al 31 de diciembre

\begin{tabular}{|c|c|c|c|}
\hline & Ventas & & $\$ 1^{\prime} 000,000.00$ \\
\hline$(-)$ & Costo de producción & & $\$ 750,000.00$ \\
\hline$(=)$ & Rendimiento bruto & & $\$ 250,000.00$ \\
\hline$(-)$ & Gastos de operación & & $\$ 44,500.00$ \\
\hline & Gastos de administración & $\$ 23,000.00$ & \\
\hline & Gastos de ventas & $21,500.00$ & \\
\hline$(=)$ & Rendimiento de operación & & $\$ 205,500.00$ \\
\hline$(\mathrm{x})$ & & $\begin{array}{l}\text { Reserva de educa- } \\
\text { ción cooperativa } 5 \%\end{array}$ & $\$ 10,275.00$ \\
\hline$(=)$ & Rendimiento antes de ISR & & $\$ 195,225.00$ \\
\hline & & ISR Artículo $177^{6}$ & $\$ 27,882.09$ \\
\hline$(=)$ & Rendimiento neto & & $\$ 167,342.91$ \\
\hline
\end{tabular}

7 DOF Disposiciones transitorias para el ejercicio fiscal 2013. 


\section{Procedimiento de cálculo del ISR Artículo 177}

\begin{tabular}{clr}
\hline $\begin{array}{c}\text { Sociedad } \\
\text { Cooperativa X } \\
(-)\end{array}$ & Rendimiento antes de ISR & $\$ 195,225.00$ \\
$(=)$ & Límite inferior & $\$ 123,580.21$ \\
$(\mathrm{x})$ & Porcedente del límite inferior & $\$ 71,644.79$ \\
$(=)$ & Impuesto marginal & $\underline{20.65 \%}$ \\
$(+)$ & Cuota fija & $\$ 14,794.65$ \\
$(=)$ & Impuesto determinado & $\$ 13,087.44$ \\
\hline
\end{tabular}

Factor de distribución: \$167,342.91 $0.2231239 \quad \$ 750,000.00$

\begin{tabular}{ccrcr}
\hline Socio & \% De ventas & $\begin{array}{c}\text { Ventas } \\
\text { realizadas }\end{array}$ & Factor & $\begin{array}{r}\text { Rendimiento } \\
\text { proporcional }\end{array}$ \\
\hline Socio A & $32 \%$ & $\$ 240,000.00$ & $22.31239 \%$ & $\$ 53,549.74$ \\
Socio B & $20 \%$ & $\$ 150,000.00$ & $22.31239 \%$ & $\$ 33,468.58$ \\
Socio C & $18 \%$ & $\$ 135,000.00$ & $22.31239 \%$ & $\$ 30,121.72$ \\
Socio D & $12 \%$ & $\$ 90,000.00$ & $22.31239 \%$ & $\$ 20,081.15$ \\
Socio E & $18 \%$ & $\$ 135,000.00$ & $22.31239 \%$ & $\$ 30,121.72$ \\
& & $\$ 750,000.00$ & & $\$ 167,342.91$ \\
\hline
\end{tabular}

\section{Tratamiento fiscal para los socios que integran una sociedad cooperativa de producción}

Capítulo I del Título IV Artículo 110 de la Ley del Impuesto Sobre la Renta

\section{Sociedad Cooperativa X}

\begin{tabular}{lrlr}
\hline Socio & Base gravable & ISR & Impuesto determinado \\
\hline Socio A & $\$ 53,549.74$ & Artículo 113 & $\$ 12,008.80$ \\
Socio B & $\$ 33,468.58$ & Artículo 113 & $\$ 6,185.26$ \\
Socio C & $\$ 30,121.72$ & Artículo 113 & $\$ 5,378.64$ \\
Socio D & $\$ 20,081.15$ & Artículo 113 & $\$ 3,110.77$ \\
Socio E & $\$ 30,121.72$ & Artículo 113 & $\$ 5,378.64$ \\
\hline
\end{tabular}


Procedimiento de cálculo del ISR Artículo $113^{8}$

\begin{tabular}{|c|c|c|}
\hline Socio A & Ingresos asimilados & $\$ 53,549.74$ \\
\hline$(-)$ & Límite inferior & $\$ 32,736.84$ \\
\hline$(=)$ & Excedente del límite inferior & $\$ 20,812.90$ \\
\hline$(\mathrm{x})$ & Porcentaje de excedente & $\underline{29} \%$ \\
\hline$(=)$ & Impuesto marginal & $\$ 6,035.74$ \\
\hline$(+)$ & Cuota fija & $\$ 5,973.06$ \\
\hline$(=)$ & Impuesto determinado & $\$ 12,008.80$ \\
\hline Socio B & Ingresos asimilados & $\$ 33,468.58$ \\
\hline$(-)$ & Límite inferior & $\$ 32,736.84$ \\
\hline$(=)$ & Excedente del límite inferior & $\$ 731.74$ \\
\hline$(\mathrm{x})$ & Porcentaje de excedente & $29 \%$ \\
\hline$(=)$ & Impuesto marginal & $\$ 212.20$ \\
\hline$(+)$ & Cuota fija & $\$ 5,973.06$ \\
\hline$(=)$ & Impuesto determinado & $\$ 6,185.26$ \\
\hline Socio $C$ & Ingresos asimilados & $\$ 30,121.72$ \\
\hline$(-)$ & Límite inferior & $\$ 20,770.30$ \\
\hline$(=)$ & Excedente del límite inferior & $\$ 9,351.42$ \\
\hline$(\mathrm{x})$ & Porcentaje de excedente & $22.73 \%$ \\
\hline$(=)$ & Impuesto marginal & $\$ 2,125.57$ \\
\hline$(+)$ & Cuota fija & $\$ 3,253.07$ \\
\hline$(=)$ & Impuesto determinado & $\$ 5,378.64$ \\
\hline Socio $D$ & Ingresos asimilados & $\$ 20,081.15$ \\
\hline$(-)$ & Límite inferior & $\$ 10,298.36$ \\
\hline$(=)$ & Excedente del límite inferior & $\$ 9,782.79$ \\
\hline$(\mathrm{x})$ & Porcentaje de excedente & $\underline{20.65 \%}$ \\
\hline$(=)$ & Impuesto marginal & $\$ 2,020.15$ \\
\hline$(+)$ & Cuota fija & $\$ 1,090.62$ \\
\hline$(=)$ & Impuesto determinado & $\$ 3,110.77$ \\
\hline Socio E & Ingresos asimilados & $\$ 30,121.72$ \\
\hline$(-)$ & Límite inferior & $\$ 20,770.30$ \\
\hline$(=)$ & Excedente del límite inferior & $\$ 9,351.42$ \\
\hline$(\mathrm{x})$ & Porcentaje de excedente & $\underline{22.73 \%}$ \\
\hline$(=)$ & Impuesto marginal & $\$ 2,125.57$ \\
\hline$(+)$ & Cuota fija & $\$ 3,253.07$ \\
\hline$(=)$ & Impuesto determinado & $\$ 5,378.64$ \\
\hline
\end{tabular}

8 DOF Disposiciones transitorias para el ejercicio fiscal 2013. 


\section{Régimen económico de las sociedades cooperativas}

Me permito citar de manera textual el apartado correspondiente a este tema, y que se encuentra contenido en la ley General de Sociedades Cooperativas; por considerarlo importante para el efecto de justificar la forma en que se explicó en el punto número 2 (dos), el tratamiento fiscal de las Sociedades Cooperativas en México; referente a la determinación de los rendimientos obtenidos y al porcentaje que se señaló como fondo de reserva de educación.

Artículo 49.-El capital de las sociedades cooperativas se integrará con las aportaciones de los socios y con los rendimientos que la Asamblea General acuerde se destinen para incrementarlo, además de considerar lo establecido en el artículo 63 de esta Ley.

Artículo 50.-Las aportaciones podrán hacerse en efectivo, bienes derechos o trabajo; estarán representadas por certificados que serán nominativos, indivisibles y de igual valor, las cuales deberán actualizarse anualmente.

La valorización de las aportaciones que no sean en efectivo, se hará en las bases constitutivas o al tiempo de ingresar el socio por acuerdo entre éste y el Consejo de Administración, con la aprobación de la Asamblea General en su momento.

El socio podrá transmitir los derechos patrimoniales que amparan sus certificados de aportación, en favor del beneficiario que designe para el caso de su muerte. Las bases constitutivas de la sociedad cooperativa, determinarán los requisitos para que también se le puedan conferir derechos cooperativos al beneficiario.

Artículo 51.-Cada socio deberá aportar por lo menos el valor de un certificado. Se podrá pactar la suscripción de certificados excedentes o voluntarios por los cuales se percibirá el interés que fije el Consejo de Administración de acuerdo con las posibilidades económicas de la sociedad cooperativa, tomando como referencia las tasas que determinen los bancos para depósitos a plazo fijo.

Al constituirse la sociedad cooperativa o al ingresar el socio a ella, será obligatoria la exhibición del 10\% cuando menos, del valor de los certificados de aportación.

Artículo 52.-Cuando la Asamblea General acuerde reducir el capital que se juzgue excedente, se hará la devolución a los socios que posean mayor número de certificados de aportación o a prorrata si todos son poseedores de un número igual de certificados. Cuando el acuerdo sea en el sentido de aumentar el capital, todos los socios quedarán obligados a suscribir el aumento en la forma y términos que acuerde la Asamblea General. 
Artículo 53.-Las sociedades cooperativas podrán constituir los siguientes fondos sociales:

I. De Reserva;

II. De Previsión Social, y

III. De Educación Cooperativa.

Artículo 54.-El Fondo de Reserva se constituirá con el 10 al $20 \%$ de los rendimientos que obtengan las sociedades cooperativas en cada ejercicio social.

Artículo 55.-El Fondo de Reserva podrá ser delimitado en las bases constitutivas, pero no será menor del $25 \%$ del capital social en las sociedades cooperativas de productores y del $10 \%$ en las de consumidores. Este fondo podrá ser afectado cuando lo requiera la sociedad para afrontar las pérdidas o restituir el capital de trabajo, debiendo de ser reintegrado al final del ejercicio social, con cargo a los rendimientos.

Artículo 55 Bis.-En las Sociedades Cooperativas de Ahorro y Préstamo, el fondo de reserva deberá constituirse por lo menos con el diez por ciento de los excedentes, que se obtengan en cada ejercicio social, hasta alcanzar un monto equivalente a, por lo menos, el diez por ciento de los activos totales de la Sociedad. Este fondo podrá ser afectado, previa decisión de la Asamblea General, cuando lo requiera la Sociedad para afrontar las pérdidas o restituir el capital de trabajo, debiendo de ser reintegrado en ejercicios subsecuentes, con cargo a los excedentes. Se entenderá por capital de trabajo a la diferencia entre activos y pasivos a plazo menor de un año.

Artículo adicionado DOF 13-08-2009.

Artículo 56.-El Fondo de Reserva de las sociedades cooperativas será manejado por el Consejo de Administración con la aprobación del Consejo de Vigilancia y podrá disponer de él, para los fines que se consignan en el artículo anterior.

Artículo 57.-El Fondo de Previsión Social no podrá ser limitado; deberá destinarse a reservas para cubrir los riesgos y enfermedades profesionales y formar fondos de pensiones y haberes de retiro de socios, primas de antigüedad y para fines diversos que cubrirán: gastos médicos y de funeral, subsidios por incapacidad, becas educacionales para los socios o sus hijos, guarderías infantiles, actividades culturales y deportivas y otras prestaciones de previsión social de naturaleza análoga. Al inicio de cada ejercicio, la Asamblea General fijará las prioridades para la aplicación de este Fondo de conformidad con las perspectivas económicas de la sociedad cooperativa. 
Las prestaciones derivadas del Fondo de Previsión Social, serán independientes de las prestaciones a que tengan derecho los socios por su afiliación a los sistemas de seguridad social.

Las sociedades cooperativas en general, deberán de afiliar obligatoriamente a sus trabajadores, y socios que aporten su trabajo personal, a los sistemas de seguridad social, e instrumentar las medidas de seguridad e higiene en el trabajo, así como de capacitación y adiestramiento, gozando del beneficio expresado en los artículos 116 y 179 de la Ley del Seguro Social.

Artículo 58.-El Fondo de Previsión Social se constituirá con la aportación anual del porcentaje, que sobre los ingresos netos, sea determinado por la Asamblea General y se aplicará en los términos del artículo anterior. Este porcentaje podrá aumentarse según los riesgos probables y la capacidad económica de la sociedad cooperativa.

Artículo 59.-El Fondo de Educación Cooperativa será constituido con el porcentaje que acuerde la Asamblea General, pero en todo caso dicho porcentaje no será inferior al $1 \%$ de los excedentes netos del mes.

Artículo reformado DOF 04-06-2001.

Artículo 60.-Las sociedades cooperativas, podrán recibir de personas físicas y morales, públicas o privadas, nacionales o internacionales, donaciones, subsidios, herencias y legados para aumentar su patrimonio.

Artículo 61.-Los excedentes de cada ejercicio social anual son la diferencia entre activo y pasivo menos la suma del capital social, las reservas y los rendimientos acumulados de años anteriores, los cuales se consignarán en el balance anual que presentará el Consejo de Administración a la Asamblea General. Igual procedimiento se observará si el balance mencionado reporta pérdidas.

Artículo 62.-Cada año las sociedades cooperativas podrán revaluar sus activos, en los términos legales correspondientes. La Asamblea General determinará con relación a los incrementos, el porcentaje que se destinará al incremento al capital social y el que se aplicará a las reservas sociales.

Artículo 63.-Las sociedades cooperativas podrán emitir certificados de aportación para capital de riesgo por tiempo determinado. 


\section{$\mathrm{VI}$. Funcionamiento y administración de las sociedades cooperativas en México}

De acuerdo con la ley General de Sociedades Cooperativas; la dirección, administración y vigilancia interna de las Sociedades Cooperativas, en general, estará a cargo de los órganos siguientes?:

— La Asamblea General;

- El Consejo de Administración;

- El Consejo de Vigilancia;

- Las comisiones y comités que esta Ley establece y las demás que designe la Asamblea General, y

- Tratándose de las Sociedades Cooperativas de Ahorro y Préstamo, además de los citados órganos, en las fracciones I a IV anteriores, deberán contar, cuando menos con:

- Comité de Crédito o su equivalente;

- Comité de Riesgos;

- Un director o gerente general, y

- Un auditor Interno.

Cada uno de los órganos que llevan a cabo la dirección, administración y vigilancia interna de las sociedades cooperativas deben de cumplir con las funciones que al efecto señale la propia Ley General de Sociedades Cooperativas, y en lo no contemplado por esta se estarán a lo dispuesto por la ley General de Sociedades Mercantiles que se encuentre vigente al momento de llevar a cabo el desempeño de sus funciones.

Para ser consejero la Ley exige como requisitos los siguientes ${ }^{10}$ :

- Acreditar la experiencia y los conocimientos mínimos que en materia financiera y administrativa, establezca la propia Cooperativa en sus bases constitutivas;

- No desempeñar simultáneamente otro cargo como dirigente, funcionario o empleado en la Cooperativa de que se trate, así como en otras Cooperativas distintas a los Organismos de Integración;

- No estar inhabilitado para ejercer el comercio;

- No estar sentenciado por delitos intencionales patrimoniales;

— No tener litigio pendiente con la Cooperativa;

\footnotetext{
9 Artículo 34 LGSC.

10 Artículo 43 Bis. LGSC.
} 
- No haber celebrado con la Cooperativa, directa o indirectamente, contratos de obras, servicios, suministros o cualquier otro de naturaleza análoga, o participar en empresas con las que la Cooperativa celebre cualquiera de los actos antes señalados;

- No desempeñar un cargo público de elección popular o de dirigencia partidista;

- No estar inhabilitado para ejercer cualquier cargo, comisión o empleo en el servicio público federal, estatal o municipal, o en el Sistema Financiero Mexicano;

- No tener parentesco por consanguinidad hasta el primer grado, afinidad hasta el segundo grado, o civil con el director o gerente general, o con alguno de los miembros del Consejo de Administración o de vigilancia de la Cooperativa, y

- Los demás que esta Ley, la asamblea o las bases constitutivas de la Cooperativa determinen.

La Asamblea General deberá conocer el perfil de los candidatos a desempeñarse como consejeros, y se someterá a su consideración la documentación e información que al efecto determine la misma Asamblea en las bases constitutivas, para evaluar la honorabilidad, historial crediticio y experiencia de negocios de los candidatos.

De acuerdo con la propuesta de reformas para el ejerció fiscal dos mil catorce (2014), que se señala a continuación, se pretende la eliminación de este régimen de personas morales que si bien de acuerdo con el Título II de la Ley del ISR, se encuentran dentro del grupo de personas morales, no tributan como tal, como ha quedado explicado de manera detallada en los apartados dos y tres del presente estudio, por lo que a manera de conclusión considero una reforma acertada por parte del Ejecutivo, al señalar que desaparecerá este régimen preferencial de las Sociedades Cooperativas de Producción, para incorporarse como realmente se constituyen dentro de la ley del Impuesto Sobre la Renta; y su régimen fiscal sea el que aplica a las personas morales de régimen general con todo el tratamiento fiscal que ello conlleva.

Régimen de Sociedades Cooperativas de Producción. ${ }^{11}$

A través de los tratamientos preferenciales se busca alentar y promover determinados sectores o actividades económicas. No obstante, la evidencia indica claramente que éstos pueden resultar ineficientes en el logro de sus objetivos, debido a que la pérdida en bienestar social generalmente supera ampliamente el beneficio otorgado. Así, las

11 Proyecto de Reformas Fiscales a la Ley de ISR, 08-09-2013. 
pérdidas recaudatorias que causan estos regímenes se dan sin que los fines extrafiscales para los que fueron diseñados se alcancen.

Además, dichos regímenes generan distorsiones en las decisiones de los agentes económicos, reducen la base gravable y la recaudación, hacen compleja la fiscalización, provocan inequidad entre los contribuyentes y crean espacios para la evasión y elusión fiscales.

El régimen aplicable a las sociedades cooperativas de producción constituidas únicamente por socios personas físicas y cuya vigencia inició desde 2006, permite que la sociedad cooperativa no pague el ISR como una persona moral sino que calcula el ISR de cada uno de sus socios, determinando la parte de la utilidad gravable del ejercicio que le corresponda a cada cooperativista por su participación en la sociedad cooperativa, difiriendo el pago del ISR hasta el ejercicio fiscal en que se distribuye la utilidad. Esta situación provoca inequidad respecto de otras empresas que por el hecho de tener una organización jurídica diferente, deben pagar el ISR cuando se generan las utilidades y no cuando se distribuyen.

Asimismo, las sociedades cooperativas de producción tienen la ventaja de efectuar anticipos a sus miembros personas físicas, los cuales son gravados como salarios con la tarifa progresiva. Dichos anticipos se disminuyen de la utilidad fiscal que determina la sociedad cooperativa permitiendo que ésta sea manipulable.

El régimen preferencial aplicable a las sociedades cooperativas de producción, dificulta las tareas de fiscalización, al impedir realizar un cruce adecuado de información con los proveedores y clientes de la misma, dado los diferentes momentos de determinación y entero del impuesto a lo largo de ejercicios fiscales distintos a aquél en que se realizaron las operaciones. De esta manera, se generan espacios para la evasión y elusión fiscales.

Por las razones expuestas, se propone eliminar el tratamiento preferencial para este sector de contribuyentes, con lo cual se pretende simplificar el ISR, evitar la inequidad existente respecto de los diversos sectores económicos y la distorsión, así como para ampliar la base del impuesto. De esta manera, estos contribuyentes se incorporarían a tributar en el régimen general de personas morales, con lo que se garantizará una contribución equitativa de todos los sectores de la economía al financiamiento de los programas públicos.

\section{Conclusiones}

Unas vez realizado el presente estudio sobre la Fiscalidad de las Cooperativas en México, se concluye que con la reforma que se presenta y que entrara en vigor a partir del próximo ejercicio fiscal, estas Sociedades Cooperativas van a tener un verdadero régimen fiscal que 
las regule, debido a que sus actividades son propias del régimen general de personas morales y que las mismas deben de aplicar para el pago del impuesto del ejercicio que corresponda, lo estipulado por el Artículo 10 de la propia Ley del Impuesto Sobre la Renta que se encuentra en vigor, y no así por lo dispuesto en el Artículo 177 que regula la forma de realizar el pago del impuesto como si se tratare del régimen de personas físicas que regula el Capítulo II del Título IV de la Ley de referencia; debiendo ser el cálculo del impuesto a pagar el siguiente:

\section{Sociedad Cooperativa $X$ \\ Estado de Rendimientos al 31 de diciembre}

\begin{tabular}{|c|c|c|c|}
\hline & Ventas & & $\$ 1^{\prime} 000,000.00$ \\
\hline$(-)$ & Costo de producción & & $\$ 750,000.00$ \\
\hline$(=)$ & Rendimiento bruto & & $\$ 250,000.00$ \\
\hline$(-)$ & Gastos de operación & & $\$ 44,500.00$ \\
\hline & Gastos de administración & $\$ 23,000.00$ & \\
\hline & Gastos de ventas & $21,500.00$ & \\
\hline$(=)$ & Rendimiento de operación & & $\$ 205,500.00$ \\
\hline$(\mathrm{x})$ & & $\begin{array}{l}\text { Reserva de educación } \\
\text { cooperativa } 5 \%\end{array}$ & $\$ 10,275.00$ \\
\hline$(=)$ & Rendimiento antes de ISR & & $\$ 195,225.00$ \\
\hline & & ISR Artículo $10^{11}$ & $\$ 56,615.25$ \\
\hline$(=)$ & Rendimiento neto & & $\$ 138,609.75$ \\
\hline
\end{tabular}

Glosario

DOF Diario Oficial de la Federación

LGSC Ley General de Sociedades Cooperativas

ISR Impuesto Sobre la Renta

\section{Bibliografía}

Código Fiscal de la Federación, Editorial ISEF, 2013.

Ley del Impuesto Sobre la Renta, Editorial ISEF, 2013.

Ley General de Sociedades Cooperativas, Última reforma DOF 13-08-09.

12 DOF Disposiciones transitorias para el ejercicio fiscal 2013. 
Ley General de Sociedades Mercantiles, Editorial ISEF, 2013.

Proyecto de Reformas Fiscales para el Ejercicio Fiscal 2014.

Páginas web

www.diputados.gob.mx

www.dof.gob.mx

www.sat.gob.mx 


\section{Derechos de autor}

La revista Deusto Estudios Cooperativos es una revista de acceso abierto lo que significa que es de libre acceso en su integridad inmediatamente después de la publicación de cada número. Se permite su lectura, la búsqueda, descarga, distribución y reutilización legal en cualquier tipo de soporte sólo para fines no comerciales y según lo previsto por la ley; sin la previa autorización de la Editorial (Universidad de Deusto) o el autor, siempre que la obra original sea debidamente citada (número, año, páginas y DOI si procede) y cualquier cambio en el original esté claramente indicado.

\section{Copyright}

The Deusto Journal of Cooperative Studies is an Open Access journal which means that it is free for full and immediate access, reading, search, download, distribution, and lawful reuse in any medium only for non-commercial purposes, without prior permission from the Publisher or the author; provided the original work is properly cited and any changes to the original are clearly indicated. 